\title{
Exergy and exergoeconomic analyses of a by- product gas-based combined cycle power plant with an air blade cooling
}

\author{
Xinyang Liu ${ }^{\mathrm{a}}$, Feng Liu ${ }^{\mathrm{a}}$, Zhaoyi Huo ${ }^{\mathrm{a},{ }^{*}}$, Qi Zhang ${ }^{\mathrm{b}}$ \\ a. School of Materials and Metallurgy, University of Science and Technology Liaoning \\ b. State Environmental Protection Key Laboratory of Eco-Industry, Northeastern University
}

\section{Contains}

Table S1 Thermodynamic properties of each flow in the 15-15 model.

Table S2 Thermodynamic properties of each flow in the 15-25 model.

Table S3 Thermodynamic properties of each flow in the 25-25 model.

Table S4 The Fuel-Product Table (15-15 model).

Table S5 The Fuel-Product Table (15-25 model).

Table S6 The Fuel-Product Table (25-25 model). 
Table S1 Thermodynamic properties of each flow in the 15-15 model.

\begin{tabular}{|c|c|c|c|c|c|c|}
\hline No. & $\begin{array}{c}T \\
\left({ }^{\circ} \mathrm{C}\right)\end{array}$ & $\begin{array}{c}P \\
(\mathrm{kPa})\end{array}$ & $\begin{array}{c}m \\
(\mathrm{~kg} / \mathrm{s})\end{array}$ & $\begin{array}{c}h \\
(\mathrm{~kJ} / \mathrm{kg})\end{array}$ & $\begin{array}{c}s \\
(\mathrm{~kJ} / \mathrm{kg}-\mathrm{K})\end{array}$ & $\begin{array}{c}\text { Exergy } \\
(\mathrm{kW})\end{array}$ \\
\hline 1 & 15 & 101.1 & 369.45 & -17.05 & 5.21 & 0 \\
\hline 2 & 237 & 601.8 & 10.03 & 210.54 & 5.28 & 2075.24 \\
\hline 3 & 322.36 & 981.5 & 26.61 & 300.90 & 5.31 & 7733.43 \\
\hline 4 & 420.18 & 1601 & 25.59 & 406.44 & 5.33 & 9966.35 \\
\hline 5 & 420.2 & 1601 & 24.43 & 406.44 & 5.33 & 9513.52 \\
\hline 6 & 420.2 & 1601 & 281.16 & 406.44 & 5.33 & 109503.78 \\
\hline 7 & 200 & 1601 & 15.53 & 170.91 & 4.92 & 4215.67 \\
\hline 8 & 200 & 1601 & 6.16 & 170.91 & 4.92 & 1673.76 \\
\hline 9 & 200 & 1601 & 2.35 & 170.91 & 4.92 & 636.73 \\
\hline 10 & 35 & 109.1 & 108.94 & -3420.20 & 5.65 & 580.69 \\
\hline 11 & 408.7 & 1595 & 108.94 & -3000.00 & 5.77 & 42460.21 \\
\hline 12 & 1260 & 1579 & 390.09 & -539.40 & 6.36 & 437385.77 \\
\hline 13 & 1211 & 1579 & 415.68 & -481.17 & 6.31 & 445902.46 \\
\hline 14 & 1039 & 802.5 & 415.68 & -698.72 & 6.35 & 351643.21 \\
\hline 15 & 1012 & 802.5 & 431.21 & -667.41 & 6.32 & 353124.25 \\
\hline 16 & 974.9 & 802.5 & 457.82 & -611.12 & 6.28 & 358475.08 \\
\hline 17 & 824.4 & 407.7 & 457.82 & -793.83 & 6.31 & 270588.10 \\
\hline 18 & 820.9 & 407.7 & 463.99 & -781.01 & 6.30 & 270321.85 \\
\hline 19 & 809.7 & 407.7 & 474.02 & -760.03 & 6.29 & 271343.12 \\
\hline 20 & 675.8 & 207.1 & 474.02 & -917.67 & 6.32 & 192218.36 \\
\hline 21 & 673.5 & 207.1 & 476.36 & -912.31 & 6.32 & 192311.92 \\
\hline 22 & 548.6 & 105.2 & 476.36 & -1049.87 & 6.35 & 122359.61 \\
\hline 23 & 540.2 & 105.2 & 476.36 & -1057.48 & 6.34 & 120006.41 \\
\hline 24 & 468.1 & 105.2 & 476.36 & -1140.44 & 6.24 & 95079.70 \\
\hline 25 & 306.6 & 105.2 & 476.36 & -1321.83 & 5.96 & 46444.98 \\
\hline 26 & 301.9 & 105.2 & 476.36 & -1326.61 & 5.95 & 45302.45 \\
\hline 27 & 242.3 & 105.2 & 476.36 & -1390.34 & 5.84 & 30981.06 \\
\hline 28 & 192.6 & 105.2 & 476.36 & -1443.81 & 5.73 & 20462.31 \\
\hline 29 & 122.1 & 105.2 & 476.36 & -1509.24 & 5.58 & 9982.56 \\
\hline 30 & 531 & 7610 & 59.35 & -12491.17 & -2.58 & 89528.38 \\
\hline 31 & 270.9 & 1020 & 59.35 & -12982.62 & -2.43 & 57758.99 \\
\hline 32 & 285 & 1020 & 9.51 & -12952.10 & -2.37 & 9398.51 \\
\hline 33 & 272.9 & 1020 & 68.87 & -12978.41 & -2.42 & 67155.82 \\
\hline 34 & 36.16 & 6 & 68.87 & -13695.52 & -2.04 & 10286.10 \\
\hline 35 & 36.16 & 6 & 68.87 & -15819.44 & -8.91 & 209.66 \\
\hline 36 & 36.36 & 1870 & 68.87 & -15816.94 & -8.91 & 341.78 \\
\hline 37 & 56.04 & 1870 & 68.87 & -15734.73 & -8.65 & 895.92 \\
\hline 38 & 67.65 & 1870 & 77.32 & -15686.17 & -8.50 & 1532.32 \\
\hline 39 & 161 & 1870 & 8.45 & -15290.38 & -7.48 & 1015.65 \\
\hline 40 & 161 & 1870 & 77.32 & -15290.38 & -7.48 & 9292.92 \\
\hline 41 & 161 & 1870 & 68.87 & -15290.38 & -7.48 & 8277.26 \\
\hline 42 & 182.4 & 1060 & 59.35 & -15197.14 & -7.27 & 9049.79 \\
\hline 43 & 184.1 & 8430 & 0.06 & -15186.03 & -7.26 & 9.71 \\
\hline 44 & 184.1 & 8430 & 59.29 & -15186.03 & -7.26 & 9594.95 \\
\hline 45 & 291.4 & 8430 & 59.29 & -14674.05 & -6.26 & 22838.92 \\
\hline
\end{tabular}




\begin{tabular}{|c|c|c|c|c|c|c|}
\hline 46 & 182.4 & 1060 & 9.51 & -13191.63 & -2.86 & 8469.95 \\
\hline 47 & 297.2 & 8260 & 59.29 & -13216.16 & -3.70 & 65621.51 \\
\hline 48 & 507 & 7660 & 59.29 & -12549.60 & -2.65 & 87233.02 \\
\hline 49 & 505.9 & 7660 & 59.35 & -12552.87 & -2.66 & 87247.76 \\
\hline 50 & 25 & 103.1 & 3443.26 & -15907.84 & -9.20 & 0 \\
\hline 51 & 25.18 & 103.1 & 3420.30 & -15865.24 & -9.06 & 2582.69 \\
\hline \multicolumn{7}{|c|}{ Unit } \\
\hline \multicolumn{3}{|c|}{$\mathrm{W}_{1}(\mathrm{AC})$} & & & \multicolumn{2}{|c|}{152351.56} \\
\hline \multicolumn{3}{|c|}{$\mathrm{W}_{2}(\mathrm{CC})$} & & & \multicolumn{2}{|c|}{45775.90} \\
\hline \multicolumn{3}{|c|}{$\mathrm{W}_{3}$ (1th in Turbine) } & & & \multicolumn{2}{|c|}{90430.07} \\
\hline \multicolumn{3}{|c|}{$\mathrm{W}_{4}$ (2th in Turbine) } & & & \multicolumn{2}{|c|}{83648.79} \\
\hline \multicolumn{3}{|c|}{$\mathrm{W}_{5}$ (3th in Turbine) } & & & \multicolumn{2}{|c|}{74726.36} \\
\hline & \multicolumn{2}{|c|}{ (4th in Turbine) } & $\mathrm{kW}$ & & \multicolumn{2}{|c|}{65526.28} \\
\hline & \multicolumn{2}{|c|}{$\mathrm{W}_{7}(\mathrm{HP})$} & & & \multicolumn{2}{|c|}{29167.95} \\
\hline & \multicolumn{2}{|c|}{$\mathrm{W}_{8}(\mathrm{LP})$} & & & \multicolumn{2}{|c|}{49384.26} \\
\hline & \multicolumn{2}{|c|}{$\mathrm{P}_{1}$ (HFP) } & & & \multicolumn{2}{|c|}{657.82} \\
\hline & \multicolumn{2}{|c|}{$\mathrm{P}_{2}(\mathrm{CP})$} & & & \multicolumn{2}{|c|}{172.73} \\
\hline & \multicolumn{2}{|c|}{$\mathrm{W}_{\text {Top }}$} & & & \multicolumn{2}{|c|}{108417.45} \\
\hline & \multicolumn{2}{|c|}{$W_{\text {Bottom }}$} & & & \multicolumn{2}{|c|}{78552.21} \\
\hline & \multicolumn{2}{|c|}{$\mathrm{W}_{\text {Total }}$} & & & \multicolumn{2}{|c|}{186969.66} \\
\hline
\end{tabular}


Table S2 Thermodynamic properties of each flow in the 15-25 model.

\begin{tabular}{|c|c|c|c|c|c|c|}
\hline No & $\begin{array}{c}T \\
\left({ }^{\circ} \mathrm{C}\right)\end{array}$ & $\begin{array}{c}P \\
(\mathrm{kPa})\end{array}$ & $\begin{array}{c}m \\
(\mathrm{~kg} / \mathrm{s})\end{array}$ & $\begin{array}{c}h \\
(\mathrm{~kJ} / \mathrm{kg})\end{array}$ & $\begin{array}{c}s \\
(\mathrm{~kJ} / \mathrm{kg}-\mathrm{K})\end{array}$ & $\begin{array}{c}\text { Exergy } \\
(\mathrm{kW})\end{array}$ \\
\hline 1 & 15 & 101.1 & 367.43 & -17.04907 & 5.2 & 384498.46 \\
\hline 2 & 253.9 & 601.8 & 10.03 & 228.34 & 5.32 & 2128.79 \\
\hline 3 & 341.8 & 981.5 & 26.61 & 321.69 & 5.34 & 7948.51 \\
\hline 4 & 440.2 & 1601 & 25.59 & 430.64 & 5.36 & 10254.48 \\
\hline 5 & 440.2 & 1601 & 24.04 & 430.64 & 5.36 & 9631.79 \\
\hline 6 & 440.2 & 1601 & 281.16 & 430.64 & 5.36 & 112669.57 \\
\hline 7 & 200 & 1601 & 15.53 & 170.91 & 4.92 & 4237.18 \\
\hline 8 & 200 & 1601 & 6.16 & 170.91 & 4.92 & 1682.31 \\
\hline 9 & 200 & 1601 & 2.35 & 170.91 & 4.92 & 639.98 \\
\hline 10 & 35 & 109.1 & 108.94 & -3420.20 & 5.65 & 540.00 \\
\hline 11 & 408.7 & 1595 & 108.94 & -3000.00 & 5.77 & 42284.23 \\
\hline 12 & 1260 & 1579 & 390.09 & -539.40 & 6.36 & 432761.23 \\
\hline 13 & 1213 & 1579 & 415.68 & -481.17 & 6.31 & 440575.87 \\
\hline 14 & 1039 & 802.5 & 415.68 & -698.72 & 6.35 & 346183.67 \\
\hline 15 & 1012 & 802.5 & 431.21 & -667.41 & 6.32 & 347609.85 \\
\hline 16 & 974.9 & 802.5 & 457.82 & -611.12 & 6.28 & 352844.16 \\
\hline 17 & 824.2 & 407.7 & 457.82 & -793.83 & 6.31 & 264810.02 \\
\hline 18 & 816.5 & 407.7 & 463.99 & -781.01 & 6.30 & 265231.38 \\
\hline 19 & 805.2 & 407.7 & 474.02 & -760.03 & 6.29 & 266222.71 \\
\hline 20 & 671.8 & 207.1 & 474.02 & -917.67 & 6.32 & 186945.22 \\
\hline 21 & 669.6 & 207.1 & 476.36 & -912.31 & 6.32 & 187029.96 \\
\hline 22 & 550.5 & 105.2 & 476.36 & -1049.87 & 6.35 & 116923.98 \\
\hline 23 & 543.9 & 105.2 & 476.36 & -1057.48 & 6.34 & 114614.98 \\
\hline 24 & 468.1 & 105.2 & 476.36 & -1140.44 & 6.24 & 90194.99 \\
\hline 25 & 306.6 & 105.2 & 476.36 & -1321.83 & 5.96 & 42871.77 \\
\hline 26 & 301.9 & 105.2 & 476.36 & -1326.61 & 5.95 & 41768.71 \\
\hline 27 & 242.3 & 105.2 & 476.36 & -1390.34 & 5.84 & 28004.06 \\
\hline 28 & 192.6 & 105.2 & 476.36 & -1443.81 & 5.73 & 18004.61 \\
\hline 29 & 133 & 105.2 & 476.36 & -1506.33 & 5.58 & 8607.87 \\
\hline 30 & 531 & 7610 & 59.35 & -12491.17 & -2.58 & 85637.37 \\
\hline 31 & 270.9 & 1020 & 59.35 & -12982.62 & -2.43 & 53777.65 \\
\hline 32 & 285 & 1020 & 9.51 & -12952.10 & -2.37 & 8754.96 \\
\hline 33 & 272.9 & 1020 & 68.87 & -12978.41 & -2.42 & 62530.87 \\
\hline 34 & 39 & 7 & 68.87 & -13679.71 & -2.05 & 6729.43 \\
\hline 35 & 39 & 7 & 68.87 & -15807.57 & -8.87 & 87.09 \\
\hline 36 & 39.6 & 1870 & 68.87 & -15805.06 & -8.87 & 218.27 \\
\hline 37 & 58.88 & 1870 & 68.87 & -15722.86 & -8.61 & 641.73 \\
\hline 38 & 70.18 & 1870 & 77.32 & -15675.59 & -8.47 & 1149.64 \\
\hline 39 & 161 & 1870 & 8.45 & -15290.38 & -7.48 & 875.64 \\
\hline 40 & 161 & 1870 & 77.32 & -15290.38 & -7.48 & 8011.85 \\
\hline 41 & 161 & 1870 & 68.87 & -15290.38 & -7.48 & 7136.21 \\
\hline 42 & 182.4 & 1060 & 59.35 & -15197.14 & -7.27 & 7940.77 \\
\hline 43 & 184.1 & 8430 & 0.06 & -15186.03 & -7.26 & 8.58 \\
\hline 44 & 184.1 & 8430 & 59.29 & -15186.03 & -7.26 & 8483.43 \\
\hline
\end{tabular}




\begin{tabular}{|c|c|c|c|c|c|c|}
\hline 45 & 291.4 & 8430 & 59.29 & -14674.05 & -6.26 & 21133.24 \\
\hline 46 & 182.4 & 1060 & 9.51 & -13191.63 & -2.86 & 7873.30 \\
\hline 47 & 297.2 & 8260 & 59.29 & -13216.16 & -3.70 & 62400.02 \\
\hline 48 & 507 & 7660 & 59.29 & -12549.60 & -2.65 & 83389.69 \\
\hline 49 & 505.9 & 7660 & 59.35 & -12552.87 & -2.66 & 83404.71 \\
\hline 50 & 25 & 101.1 & 3420.30 & -15866 & -9.06 & 0 \\
\hline 51 & 35.18 & 101.1 & 3420.30 & -15823.44 & -8.92 & 2501.90 \\
\hline \multicolumn{7}{|c|}{ Unit } \\
\hline \multicolumn{3}{|c|}{$\mathrm{W}_{1}(\mathrm{AC})$} & & & \multicolumn{2}{|c|}{152351.561} \\
\hline \multicolumn{3}{|c|}{$\mathrm{W}_{2}(\mathrm{CC})$} & & & \multicolumn{2}{|c|}{45775.90} \\
\hline \multicolumn{3}{|c|}{$\mathrm{W}_{3}$ (1th in Turbine) } & & & \multicolumn{2}{|c|}{90430.067} \\
\hline \multicolumn{3}{|c|}{$\mathrm{W}_{4}$ (2th in Turbine) } & & & \multicolumn{2}{|c|}{83648.79} \\
\hline \multicolumn{3}{|c|}{$\mathrm{W}_{5}$ (3th in Turbine) } & & & \multicolumn{2}{|c|}{74726.356} \\
\hline \multicolumn{3}{|c|}{$\mathrm{W}_{6}$ (4th in Turbine) } & \multicolumn{2}{|c|}{$\mathrm{kW}$} & \multicolumn{2}{|c|}{65526.28} \\
\hline \multicolumn{3}{|c|}{$\mathrm{W}_{7}(\mathrm{HP})$} & & & \multicolumn{2}{|c|}{29167.95} \\
\hline \multicolumn{3}{|c|}{$\mathrm{W}_{8}(\mathrm{LP})$} & & & \multicolumn{2}{|c|}{49384.26} \\
\hline \multicolumn{3}{|c|}{$\mathrm{P}_{1}(\mathrm{HFP})$} & & & \multicolumn{2}{|c|}{657.82} \\
\hline \multicolumn{3}{|c|}{$\mathrm{P}_{2}(\mathrm{CP})$} & & & \multicolumn{2}{|c|}{172.73} \\
\hline \multicolumn{3}{|c|}{$\mathrm{W}_{\text {Top }}$} & & & \multicolumn{2}{|c|}{108417.45} \\
\hline \multicolumn{3}{|c|}{$\mathrm{W}_{\text {Bottom }}$} & & & \multicolumn{2}{|c|}{77463.68} \\
\hline \multicolumn{3}{|c|}{$\mathrm{W}_{\text {Total }}$} & & & \multicolumn{2}{|c|}{185881.13} \\
\hline
\end{tabular}


Table S3 Thermodynamic properties of each flow in the 25-25 model.

\begin{tabular}{|c|c|c|c|c|c|c|}
\hline No & $\begin{array}{c}T \\
\left({ }^{\circ} \mathrm{C}\right)\end{array}$ & $\begin{array}{c}P \\
(\mathrm{kPa})\end{array}$ & $\begin{array}{c}m \\
(\mathrm{~kg} / \mathrm{s})\end{array}$ & $\begin{array}{c}h \\
(\mathrm{~kJ} / \mathrm{kg})\end{array}$ & $\begin{array}{c}s \\
(\mathrm{~kJ} / \mathrm{kg}-\mathrm{K})\end{array}$ & $\begin{array}{c}\text { Exergy } \\
(\mathrm{kW})\end{array}$ \\
\hline 1 & 25 & 101.1 & 357.65 & -7.008711 & 5.24 & 0.00 \\
\hline 2 & 253.9 & 601.8 & 9.81 & 228.34 & 5.32 & 2081.68 \\
\hline 3 & 341.8 & 981.5 & 26.64 & 321.69 & 5.34 & 7957.77 \\
\hline 4 & 442.4 & 1601 & 25.92 & 430.64 & 5.36 & 10386.64 \\
\hline 5 & 442.4 & 1601 & 23.53 & 430.64 & 5.36 & 9427.86 \\
\hline 6 & 442.4 & 1601 & 271.75 & 430.64 & 5.36 & 108901.11 \\
\hline 7 & 200 & 1601 & 15.72 & 170.91 & 4.92 & 4290.89 \\
\hline 8 & 200 & 1601 & 5.73 & 170.91 & 4.92 & 1563.80 \\
\hline 9 & 200 & 1601 & 2.07 & 170.91 & 4.92 & 565.89 \\
\hline 10 & 35 & 109.1 & 102.71 & -3420.20 & 5.65 & 509.11 \\
\hline 11 & 408.7 & 1595 & 102.71 & -3000.00 & 5.77 & 39865.12 \\
\hline 12 & 1260 & 1579 & 374.46 & -505.21 & 6.36 & 415245.44 \\
\hline 13 & 1212 & 1579 & 400.38 & -444.62 & 6.31 & 423667.64 \\
\hline 14 & 1038 & 802.5 & 400.38 & -662.14 & 6.34 & 332760.94 \\
\hline 15 & 1009 & 802.5 & 416.10 & -630.66 & 6.32 & 334211.60 \\
\hline 16 & 972 & 802.5 & 442.74 & -573.35 & 6.28 & 339887.64 \\
\hline 17 & 821.3 & 407.7 & 442.74 & -755.76 & 6.31 & 254880.29 \\
\hline 18 & 814 & 407.7 & 448.47 & -743.92 & 6.30 & 255275.02 \\
\hline 19 & 802.9 & 407.7 & 458.28 & -723.11 & 6.29 & 256375.45 \\
\hline 20 & 669.5 & 207.1 & 458.28 & -880.52 & 6.32 & 179832.27 \\
\hline 21 & 667.5 & 207.1 & 460.36 & -875.78 & 6.32 & 179908.15 \\
\hline 22 & 548.5 & 105.2 & 460.36 & -1013.12 & 6.35 & 112252.48 \\
\hline 23 & 541.9 & 105.2 & 460.36 & -1020.68 & 6.34 & 110040.45 \\
\hline 24 & 469.3 & 105.2 & 460.36 & -1102.87 & 6.24 & 86690.76 \\
\hline 25 & 306.5 & 105.2 & 460.36 & -1282.59 & 5.96 & 41416.20 \\
\hline 26 & 302.1 & 105.2 & 460.36 & -1287.44 & 5.95 & 40335.95 \\
\hline 27 & 243.4 & 105.2 & 460.36 & -1350.57 & 5.84 & 27150.84 \\
\hline 28 & 192.6 & 105.2 & 460.36 & -1404.52 & 5.73 & 17395.11 \\
\hline 29 & 134.7 & 105.2 & 460.36 & -1465.20 & 5.59 & 8541.67 \\
\hline 30 & 531 & 7610 & 56.83 & -12491.17 & -2.58 & 81997.68 \\
\hline 31 & 270.9 & 1020 & 56.83 & -12982.62 & -2.43 & 51492.04 \\
\hline 32 & 285 & 1020 & 9.32 & -12952.10 & -2.37 & 8574.41 \\
\hline 33 & 272.9 & 1020 & 68.87 & -12978.41 & -2.42 & 62530.87 \\
\hline 34 & 39 & 7 & 68.87 & -13679.66 & -2.05 & 6733.03 \\
\hline 35 & 39 & 7 & 68.87 & -15807.57 & -8.87 & 87.09 \\
\hline 36 & 39.2 & 1870 & 68.87 & -15805.06 & -8.87 & 218.27 \\
\hline 37 & 61.32 & 1870 & 68.87 & -15712.69 & -8.58 & 715.82 \\
\hline 38 & 72.75 & 1870 & 74.60 & -15664.85 & -8.44 & 1217.59 \\
\hline 39 & 161 & 1870 & 8.45 & -15290.38 & -7.48 & 875.64 \\
\hline 40 & 161 & 1870 & 74.60 & -15290.38 & -7.48 & 7730.12 \\
\hline 41 & 161 & 1870 & 66.15 & -15290.38 & -7.48 & 6854.48 \\
\hline 42 & 182.4 & 1060 & 56.83 & -15197.14 & -7.27 & 7603.28 \\
\hline 43 & 184.1 & 8430 & 0.06 & -15186.03 & -7.26 & 8.58 \\
\hline 44 & 184.1 & 8430 & 56.77 & -15186.03 & -7.26 & 8122.51 \\
\hline
\end{tabular}




\begin{tabular}{|c|c|c|c|c|c|c|}
\hline 45 & 291.4 & 8430 & 56.77 & -14674.05 & -6.26 & 20234.15 \\
\hline 46 & 182.4 & 1060 & 9.32 & -13191.63 & -2.86 & 7710.93 \\
\hline 47 & 297.2 & 8260 & 56.77 & -13216.16 & -3.70 & 59745.26 \\
\hline 48 & 507 & 7660 & 56.77 & -12549.60 & -2.65 & 79841.94 \\
\hline 49 & 505.9 & 7660 & 56.83 & -12552.87 & -2.66 & 79859.91 \\
\hline 50 & 25 & 101.1 & 3307.32 & -15866.00 & -9.06 & 0.00 \\
\hline 51 & 35.18 & 101.1 & 3307.32 & -15823.44 & -8.92 & 2419.26 \\
\hline \multicolumn{7}{|c|}{ Unit } \\
\hline \multicolumn{3}{|c|}{$\mathrm{W}_{1}(\mathrm{AC})$} & & & \multicolumn{2}{|c|}{153166.66} \\
\hline \multicolumn{3}{|c|}{$\mathrm{W}_{2}(\mathrm{CC})$} & & & \multicolumn{2}{|c|}{43157.03} \\
\hline \multicolumn{3}{|c|}{$\mathrm{W}_{3}$ (1th in Turbine) } & & & \multicolumn{2}{|c|}{87086.88} \\
\hline \multicolumn{3}{|c|}{$\mathrm{W}_{4}$ (2th in Turbine) } & & & \multicolumn{2}{|c|}{80762.13} \\
\hline \multicolumn{3}{|c|}{$\mathrm{W}_{5}(3$ th in Turbine $)$} & & & \multicolumn{2}{|c|}{72138.85} \\
\hline \multicolumn{3}{|c|}{$\mathrm{W}_{6}$ (4th in Turbine) } & \multicolumn{2}{|c|}{$\mathrm{kW}$} & \multicolumn{2}{|c|}{63225.66} \\
\hline \multicolumn{3}{|c|}{$\mathrm{W}_{7}(\mathrm{HP})$} & & & \multicolumn{2}{|c|}{27928.28} \\
\hline \multicolumn{3}{|c|}{$\mathrm{W}_{8}(\mathrm{LP})$} & & & \multicolumn{2}{|c|}{46391.12} \\
\hline \multicolumn{3}{|c|}{$\mathrm{P}_{1}(\mathrm{HFP})$} & & & \multicolumn{2}{|c|}{631.42} \\
\hline \multicolumn{3}{|c|}{$\mathrm{P}_{2}(\mathrm{CP})$} & & & \multicolumn{2}{|c|}{165.54} \\
\hline \multicolumn{3}{|c|}{$\mathrm{W}_{\text {Top }}$} & & & \multicolumn{2}{|c|}{98868.46} \\
\hline \multicolumn{3}{|c|}{$\mathrm{W}_{\text {Bottom }}$} & & & \multicolumn{2}{|c|}{74319.40} \\
\hline \multicolumn{3}{|c|}{$\mathrm{W}_{\text {Total }}$} & & & \multicolumn{2}{|c|}{173187.86} \\
\hline
\end{tabular}




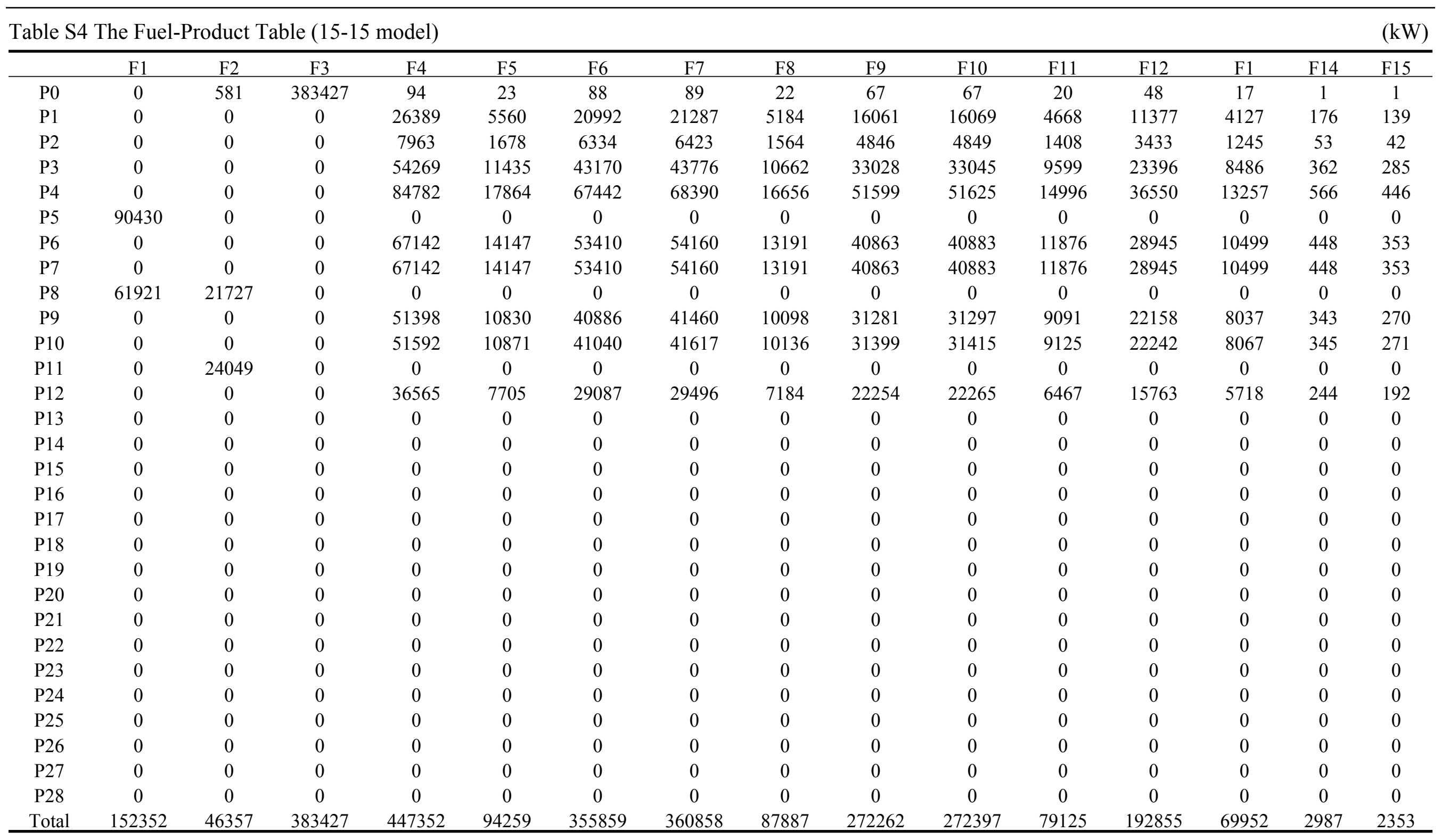




\begin{tabular}{|c|c|c|c|c|c|c|c|c|c|c|c|c|c|c|c|c|}
\hline & F16 & $\mathrm{F} 17$ & F18 & F19 & $\mathrm{F} 20$ & $\mathrm{~F} 21$ & $\mathrm{~F} 22$ & $\mathrm{~F} 23$ & $\mathrm{~F} 24$ & $\mathrm{~F} 2$ & F26 & $\mathrm{F} 27$ & $\mathrm{~F} 28$ & $\mathrm{P}$ & $\mathrm{R}$ & Total \\
\hline $\mathrm{P} 0$ & 6 & 12 & 0 & 4 & 3 & 3 & 0 & 0 & 0 & 0 & 0 & 2 & 0 & 0 & 0 & 384573 \\
\hline $\mathrm{P} 1$ & 1470 & 2869 & 67 & 845 & 621 & 618 & 0 & 0 & 0 & 0 & 0 & 589 & 0 & 0 & 0 & 138792 \\
\hline $\mathrm{P} 2$ & 444 & 866 & 20 & 255 & 187 & 187 & 0 & 0 & 0 & 0 & 0 & 178 & 0 & 0 & 0 & 41880 \\
\hline P3 & 3024 & 5900 & 139 & 1737 & 1276 & 1271 & 0 & 0 & 0 & 0 & 0 & 1211 & 0 & 0 & 0 & 285422 \\
\hline P4 & 4724 & 9217 & 217 & 2714 & 1994 & 1986 & 0 & 0 & 0 & 0 & 0 & 1892 & 0 & 0 & 0 & 445902 \\
\hline P5 & 0 & 0 & 0 & 0 & 0 & 0 & 0 & 0 & 0 & 0 & 0 & 0 & 0 & 0 & 0 & 90430 \\
\hline P6 & 3741 & 7299 & 171 & 2149 & 1579 & 1573 & 0 & 0 & 0 & 0 & 0 & 1498 & 0 & 0 & 0 & 353124 \\
\hline P7 & 3741 & 7299 & 171 & 2149 & 1579 & 1573 & 0 & 0 & 0 & 0 & 0 & 1498 & 0 & 0 & 0 & 353124 \\
\hline P8 & 0 & 0 & 0 & 0 & 0 & 0 & 0 & 0 & 0 & 0 & 0 & 0 & 0 & 0 & 0 & 83649 \\
\hline P9 & 2864 & 5588 & 131 & 1645 & 1209 & 1204 & 0 & 0 & 0 & 0 & 0 & 1147 & 0 & 0 & 0 & 270322 \\
\hline $\mathrm{P} 10$ & 2875 & 5609 & 132 & 1652 & 1213 & 1209 & 0 & 0 & 0 & 0 & 0 & 1151 & 0 & 0 & 0 & 271343 \\
\hline P11 & 0 & 0 & 0 & 0 & 0 & 0 & 0 & 0 & 0 & 0 & 0 & 0 & 42891 & 0 & 0 & 74726 \\
\hline $\mathrm{P} 12$ & 2037 & 3975 & 93 & 1171 & 860 & 857 & 0 & 0 & 0 & 0 & 0 & 816 & 0 & 0 & 0 & 192312 \\
\hline P13 & 0 & 0 & 0 & 0 & 0 & 0 & 0 & 0 & 0 & 0 & 0 & 0 & 65526 & 0 & 0 & 65526 \\
\hline $\mathrm{P} 14$ & 0 & 0 & 0 & 0 & 0 & 0 & 178 & 318 & 0 & 0 & 56 & 0 & 0 & 0 & 0 & 554 \\
\hline P15 & 0 & 0 & 0 & 0 & 0 & 0 & 731 & 1309 & 0 & 0 & 232 & 0 & 0 & 0 & 0 & 2281 \\
\hline P16 & 0 & 0 & 0 & 0 & 0 & 0 & 6929 & 12403 & 0 & 0 & 2198 & 0 & 0 & 0 & 0 & 21612 \\
\hline P17 & 0 & 0 & 0 & 0 & 0 & 0 & 13716 & 24553 & 0 & 0 & 4350 & 0 & 0 & 0 & 0 & 42783 \\
\hline P18 & 0 & 0 & 0 & 0 & 0 & 0 & 298 & 533 & 0 & 0 & 94 & 0 & 0 & 0 & 0 & 929 \\
\hline P19 & 0 & 0 & 0 & 0 & 0 & 0 & 4246 & 7601 & 0 & 0 & 1347 & 0 & 0 & 0 & 0 & 13244 \\
\hline $\mathrm{P} 20$ & 0 & 0 & 0 & 0 & 0 & 0 & 2963 & 5304 & 0 & 0 & 940 & 0 & 0 & 0 & 0 & 9242 \\
\hline $\mathrm{P} 21$ & 0 & 0 & 0 & 0 & 0 & 0 & 2488 & 4454 & 0 & 0 & 789 & 0 & 0 & 0 & 0 & 7761 \\
\hline $\mathrm{P} 22$ & 0 & 0 & 0 & 0 & 0 & 0 & 0 & 0 & 0 & 0 & 0 & 0 & 29168 & 0 & 0 & 29168 \\
\hline $\mathrm{P} 23$ & 0 & 0 & 0 & 0 & 0 & 0 & 0 & 0 & 0 & 0 & 0 & 0 & 49384 & 0 & 0 & 49384 \\
\hline $\mathrm{P} 24$ & 0 & 0 & 0 & 0 & 0 & 0 & 178 & 318 & 0 & 0 & 56 & 0 & 0 & 0 & 0 & 555 \\
\hline $\mathrm{P} 25$ & 0 & 0 & 0 & 0 & 0 & 0 & 42 & 76 & 0 & 0 & 13 & 0 & 0 & 0 & 0 & 132 \\
\hline P26 & 0 & 0 & 0 & 0 & 0 & 0 & 0 & 0 & 0 & 0 & 0 & 0 & 0 & 0 & 2583 & 2583 \\
\hline $\mathrm{P} 27$ & 0 & 0 & 0 & 0 & 0 & 0 & 0 & 0 & 0 & 0 & 0 & 0 & 0 & 0 & 9983 & 9983 \\
\hline P28 & 0 & 0 & 0 & 0 & 0 & 0 & 0 & 0 & 658 & 173 & 0 & 0 & 0 & 186139 & 0 & 186139 \\
\hline Total & 24927 & 48635 & 1143 & 14321 & 10519 & 10480 & 31769 & 56870 & 658 & 173 & 10076 & 9983 & 186970 & 186139 & 12565 & \\
\hline
\end{tabular}




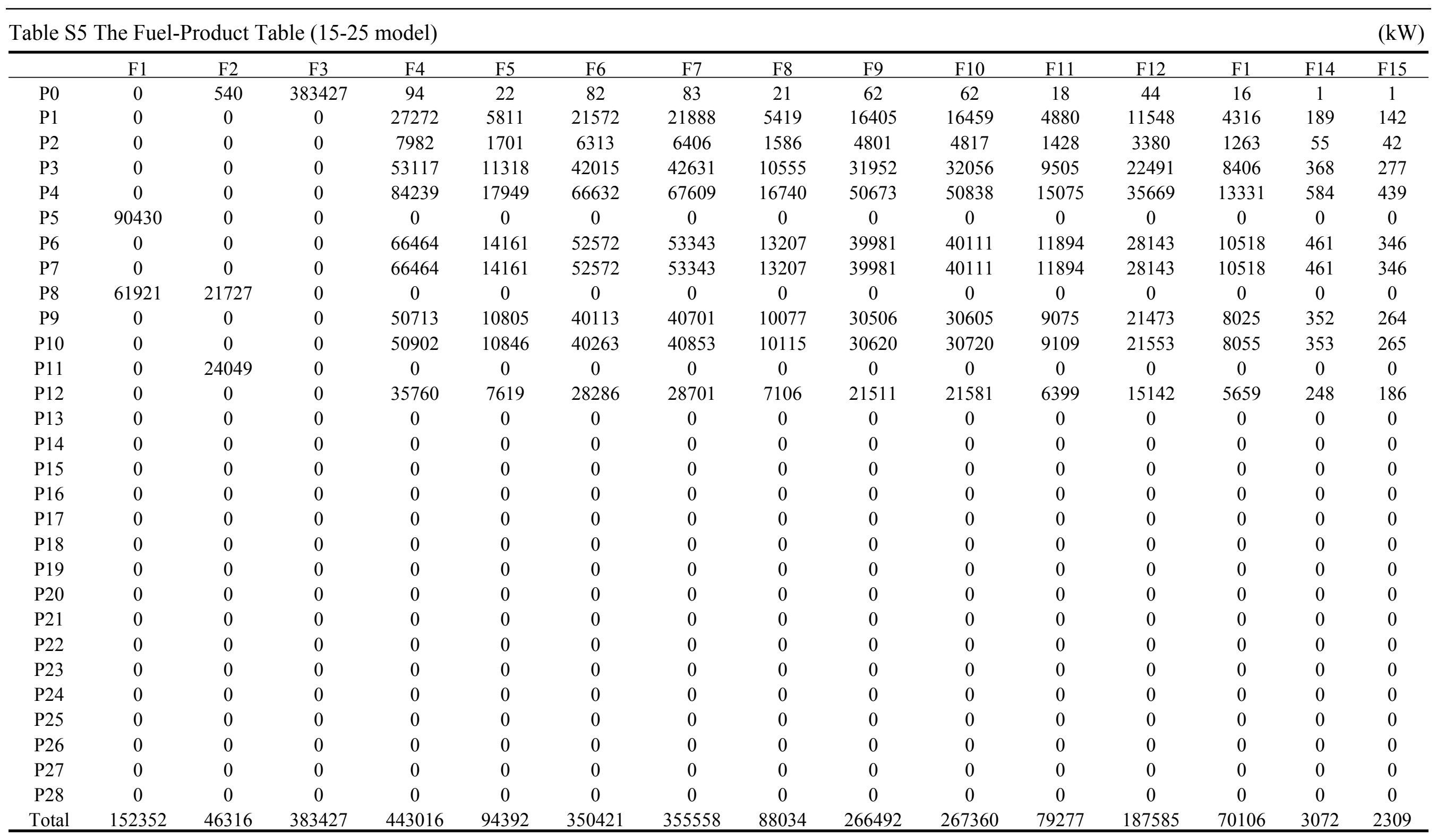




\begin{tabular}{|c|c|c|c|c|c|c|c|c|c|c|c|c|c|c|c|c|}
\hline & F16 & F17 & F18 & F19 & F20 & F21 & F22 & F23 & $\mathrm{F} 24$ & $\mathrm{~F} 2$ & F26 & F27 & F28 & $\mathrm{P}$ & $\mathrm{R}$ & Total \\
\hline $\mathrm{P} 0$ & 6 & 11 & 0 & 3 & 2 & 2 & 0 & 0 & 0 & 0 & 0 & 2 & 0 & 0 & 0 & 362507 \\
\hline $\mathrm{P} 1$ & 1503 & 2913 & 68 & 847 & 616 & 578 & 0 & 0 & 0 & 0 & 0 & 530 & 0 & 0 & 0 & 142633 \\
\hline $\mathrm{P} 2$ & 440 & 853 & 20 & 248 & 180 & 169 & 0 & 0 & 0 & 0 & 0 & 155 & 0 & 0 & 0 & 41744 \\
\hline P3 & 2928 & 5674 & 132 & 1650 & 1199 & 1127 & 0 & 0 & 0 & 0 & 0 & 1032 & 0 & 0 & 0 & 277807 \\
\hline $\mathrm{P} 4$ & 4643 & 8998 & 210 & 2617 & 1901 & 1787 & 0 & 0 & 0 & 0 & 0 & 1637 & 0 & 0 & 0 & 440576 \\
\hline P5 & 0 & 0 & 0 & 0 & 0 & 0 & 0 & 0 & 0 & 0 & 0 & 0 & 0 & 0 & 0 & 90430 \\
\hline P6 & 3664 & 7100 & 165 & 2065 & 1500 & 1410 & 0 & 0 & 0 & 0 & 0 & 1291 & 0 & 0 & 0 & 347610 \\
\hline P7 & 3664 & 7100 & 165 & 2065 & 1500 & 1410 & 0 & 0 & 0 & 0 & 0 & 1291 & 0 & 0 & 0 & 347610 \\
\hline P8 & 0 & 0 & 0 & 0 & 0 & 0 & 0 & 0 & 0 & 0 & 0 & 0 & 0 & 0 & 0 & 83649 \\
\hline P9 & 2795 & 5417 & 126 & 1576 & 1145 & 1076 & 0 & 0 & 0 & 0 & 0 & 985 & 0 & 0 & 0 & 265231 \\
\hline P10 & 2806 & 5437 & 127 & 1582 & 1149 & 1080 & 0 & 0 & 0 & 0 & 0 & 989 & 0 & 0 & 0 & 266223 \\
\hline P11 & 0 & 0 & 0 & 0 & 0 & 0 & 0 & 0 & 0 & 0 & 0 & 0 & 42891 & 0 & 0 & 74726 \\
\hline P12 & 1971 & 3820 & 89 & 1111 & 807 & 759 & 0 & 0 & 0 & 0 & 0 & 695 & 0 & 0 & 0 & 187030 \\
\hline $\mathrm{P} 13$ & 0 & 0 & 0 & 0 & 0 & 0 & 0 & 0 & 0 & 0 & 0 & 0 & 65526 & 0 & 0 & 65526 \\
\hline P14 & 0 & 0 & 0 & 0 & 0 & 0 & 143 & 250 & 0 & 0 & 30 & 0 & 0 & 0 & 0 & 423 \\
\hline P16 & 0 & 0 & 0 & 0 & 0 & 0 & 7064 & 12372 & 0 & 0 & 1473 & 0 & 0 & 0 & 0 & 20990 \\
\hline P17 & 0 & 0 & 0 & 0 & 0 & 0 & 13888 & 24325 & 0 & 0 & 2896 & 0 & 0 & 0 & 0 & 41267 \\
\hline P18 & 0 & 0 & 0 & 0 & 0 & 0 & 297 & 520 & 0 & 0 & 62 & 0 & 0 & 0 & 0 & 882 \\
\hline P19 & 0 & 0 & 0 & 0 & 0 & 0 & 4257 & 7456 & 0 & 0 & 888 & 0 & 0 & 0 & 0 & 12650 \\
\hline $\mathrm{P} 20$ & 0 & 0 & 0 & 0 & 0 & 0 & 2921 & 5115 & 0 & 0 & 609 & 0 & 0 & 0 & 0 & 8678 \\
\hline $\mathrm{P} 21$ & 0 & 0 & 0 & 0 & 0 & 0 & 2309 & 4045 & 0 & 0 & 481 & 0 & 0 & 0 & 0 & 6862 \\
\hline P22 & 0 & 0 & 0 & 0 & 0 & 0 & 0 & 0 & 0 & 0 & 0 & 0 & 29168 & 0 & 0 & 29168 \\
\hline $\mathrm{P} 23$ & 0 & 0 & 0 & 0 & 0 & 0 & 0 & 0 & 0 & 0 & 0 & 0 & 48296 & 0 & 0 & 48296 \\
\hline P24 & 0 & 0 & 0 & 0 & 0 & 0 & 186 & 325 & 0 & 0 & 39 & 0 & 0 & 0 & 0 & 551 \\
\hline $\mathrm{P} 25$ & 0 & 0 & 0 & 0 & 0 & 0 & 44 & 77 & 0 & 0 & 9 & 0 & 0 & 0 & 0 & 131 \\
\hline P26 & 0 & 0 & 0 & 0 & 0 & 0 & 0 & 0 & 0 & 0 & 0 & 0 & 0 & 0 & 2502 & 2502 \\
\hline $\mathrm{P} 27$ & 0 & 0 & 0 & 0 & 0 & 0 & 0 & 0 & 0 & 0 & 0 & 0 & 0 & 0 & 8608 & 8608 \\
\hline P28 & 0 & 0 & 0 & 0 & 0 & 0 & 0 & 0 & 659 & 172 & 0 & 0 & 0 & 185049 & 0 & 185049 \\
\hline Total & 24420 & 47323 & 1103 & 13765 & 9999 & 9397 & 31860 & 55801 & 659 & 172 & 6642 & 8608 & 185881 & 185049 & 11110 & \\
\hline
\end{tabular}




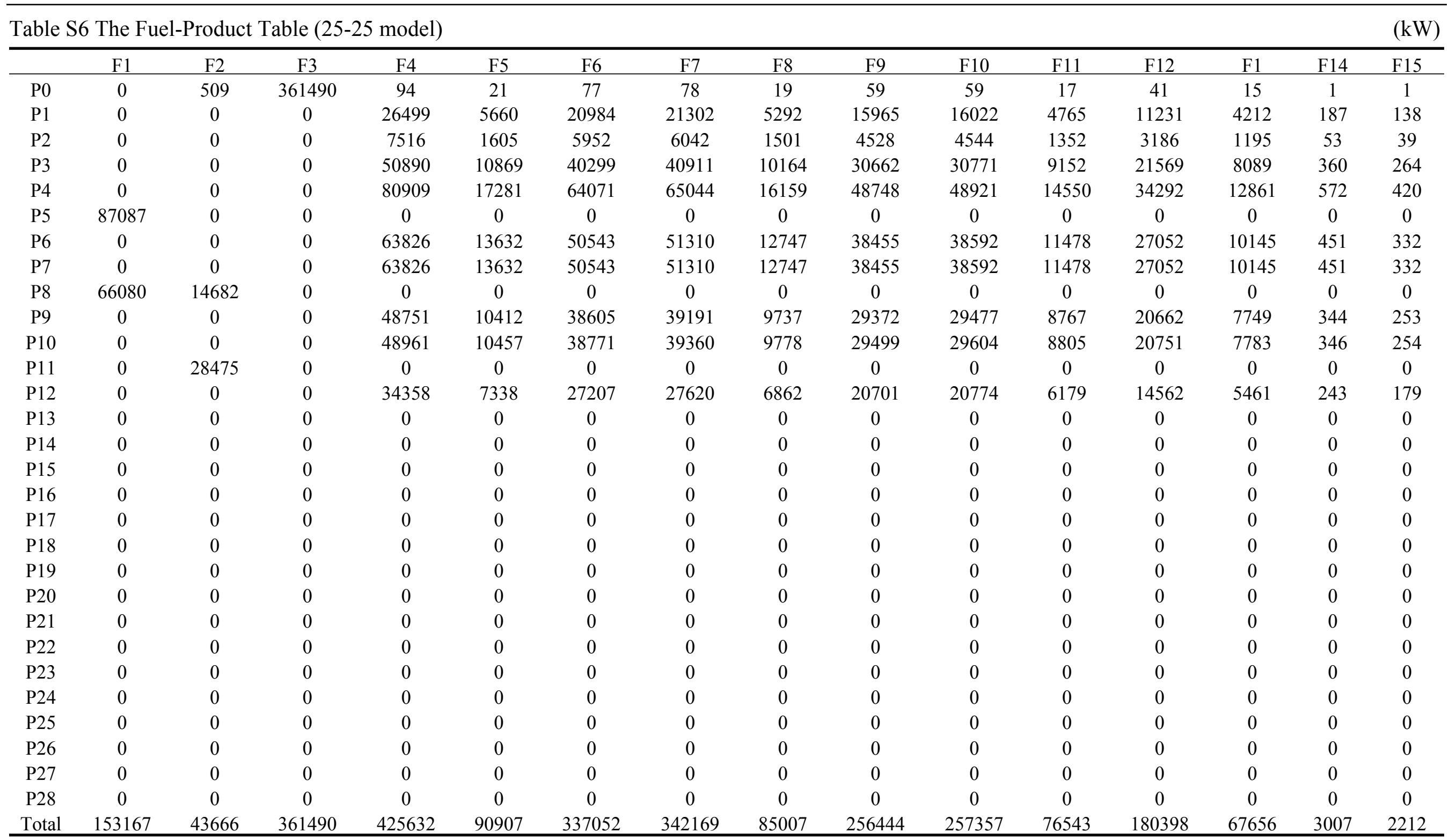




\begin{tabular}{|c|c|c|c|c|c|c|c|c|c|c|c|c|c|c|c|c|}
\hline & F16 & F17 & F18 & F19 & F20 & F21 & F22 & F23 & F24 & $\mathrm{F} 2$ & F26 & F27 & F28 & $\mathrm{P}$ & $\mathrm{R}$ & Total \\
\hline P0 & 5 & 10 & 0 & 3 & 2 & 2 & 0 & 0 & 0 & 0 & 0 & 2 & 0 & 0 & 0 & 362507 \\
\hline $\mathrm{P} 1$ & 1454 & 2819 & 67 & 821 & 607 & 551 & 0 & 0 & 0 & 0 & 0 & 532 & 0 & 0 & 0 & 138755 \\
\hline $\mathrm{P} 2$ & 412 & 799 & 19 & 233 & 172 & 156 & 0 & 0 & 0 & 0 & 0 & 151 & 0 & 0 & 0 & 39356 \\
\hline P3 & 2792 & 5413 & 129 & 1576 & 1166 & 1059 & 0 & 0 & 0 & 0 & 0 & 1021 & 0 & 0 & 0 & 266479 \\
\hline P4 & 4439 & 8606 & 205 & 2506 & 1854 & 1683 & 0 & 0 & 0 & 0 & 0 & 1624 & 0 & 0 & 0 & 423669 \\
\hline P5 & 0 & 0 & 0 & 0 & 0 & 0 & 0 & 0 & 0 & 0 & 0 & 0 & 0 & 0 & 0 & 87087 \\
\hline P6 & 3501 & 6789 & 162 & 1977 & 1463 & 1328 & 0 & 0 & 0 & 0 & 0 & 1281 & 0 & 0 & 0 & 334211 \\
\hline P7 & 3501 & 6789 & 162 & 1977 & 1463 & 1328 & 0 & 0 & 0 & 0 & 0 & 1281 & 0 & 0 & 0 & 334211 \\
\hline P8 & 0 & 0 & 0 & 0 & 0 & 0 & 0 & 0 & 0 & 0 & 0 & 0 & 0 & 0 & 0 & 80762 \\
\hline P9 & 2674 & 5186 & 124 & 1510 & 1117 & 1014 & 0 & 0 & 0 & 0 & 0 & 978 & 0 & 0 & 0 & 255275 \\
\hline P10 & 2686 & 5208 & 124 & 1517 & 1122 & 1018 & 0 & 0 & 0 & 0 & 0 & 983 & 0 & 0 & 0 & 256375 \\
\hline P11 & 0 & 0 & 0 & 0 & 0 & 0 & 0 & 0 & 0 & 0 & 0 & 0 & 35643 & 0 & 0 & 72139 \\
\hline P12 & 1885 & 3655 & 87 & 1064 & 787 & 715 & 0 & 0 & 0 & 0 & 0 & 689 & 0 & 0 & 0 & 179908 \\
\hline P13 & 0 & 0 & 0 & 0 & 0 & 0 & 0 & 0 & 0 & 0 & 0 & 0 & 63226 & 0 & 0 & 63226 \\
\hline P14 & 0 & 0 & 0 & 0 & 0 & 0 & 167 & 306 & 0 & 0 & 36 & 0 & 0 & 0 & 0 & 498 \\
\hline P16 & 0 & 0 & 0 & 0 & 0 & 0 & 6748 & 12343 & 0 & 0 & 1470 & 0 & 0 & 0 & 0 & 20097 \\
\hline P17 & 0 & 0 & 0 & 0 & 0 & 0 & 13267 & 24267 & 0 & 0 & 2890 & 0 & 0 & 0 & 0 & 39511 \\
\hline P18 & 0 & 0 & 0 & 0 & 0 & 0 & 290 & 530 & 0 & 0 & 63 & 0 & 0 & 0 & 0 & 863 \\
\hline P19 & 0 & 0 & 0 & 0 & 0 & 0 & 4067 & 7439 & 0 & 0 & 886 & 0 & 0 & 0 & 0 & 12112 \\
\hline P20 & 0 & 0 & 0 & 0 & 0 & 0 & 2841 & 5196 & 0 & 0 & 619 & 0 & 0 & 0 & 0 & 8460 \\
\hline P21 & 0 & 0 & 0 & 0 & 0 & 0 & 2187 & 4000 & 0 & 0 & 476 & 0 & 0 & 0 & 0 & 6512.53 \\
\hline P22 & 0 & 0 & 0 & 0 & 0 & 0 & 0 & 0 & 0 & 0 & 0 & 0 & 27928 & 0 & 0 & 27928 \\
\hline P23 & 0 & 0 & 0 & 0 & 0 & 0 & 0 & 0 & 0 & 0 & 0 & 0 & 46391 & 0 & 0 & 46391 \\
\hline P24 & 0 & 0 & 0 & 0 & 0 & 0 & 177 & 324 & 0 & 0 & 39 & 0 & 0 & 0 & 0 & 528 \\
\hline P25 & 0 & 0 & 0 & 0 & 0 & 0 & 44 & 81 & 0 & 0 & 10 & 0 & 0 & 0 & 0 & 131 \\
\hline P26 & 0 & 0 & 0 & 0 & 0 & 0 & 0 & 0 & 0 & 0 & 0 & 0 & 0 & 0 & 2419 & 2419 \\
\hline $\mathrm{P} 27$ & 0 & 0 & 0 & 0 & 0 & 0 & 0 & 0 & 0 & 0 & 0 & 0 & 0 & 0 & 8542 & 8542 \\
\hline P28 & 0 & 0 & 0 & 0 & 0 & 0 & 0 & 0 & 631 & 166 & 0 & 0 & 0 & 172391 & 0 & 172391 \\
\hline Total & 23350 & 45275 & 1080 & 13185 & 9756 & 8853 & 30506 & 55798 & 631 & 166 & 6646 & 8542 & 173188 & 172391 & 10961 & \\
\hline
\end{tabular}


